\title{
SEMI-ANALYTICAL SOLUTION OF THE HEAT CONDUCTION IN A PLATE WITH HEAT GENERATION
}

\author{
L. C. da Silva, \\ D. J. N. M. Chalhub, \\ A. L. O. Calil, \\ and R. S. de Moura \\ Universidade do Estado do Rio de Janeiro \\ Programa de Pós-Graduação em Engenharia \\ Mecânica - PPGEM \\ Bairro São Cristóvão \\ CEP. 20940-200, Rio de Janeiro, RJ, Brasil \\ lucascoelho.silva@ hotmail.com \\ Received: December 16, 2016 \\ Revised: January 18, 2016 \\ Accepted: April 02, 2017
}

\section{ABSTRACT}

In the present work, a formulation for the solution of the two-dimensional steady state heat conduction with heat generation is presented. The classical integral transform technique (CITT) is used to solve the problem in a semianalytical manner. CITT deals with expansions of the sought solution in terms of infinite orthogonal basis of eigenfunctions, keeping the solution process always within a continuous domain. For the particular problem, the resulting system is composed of a set of uncoupled differential equations which can be solved analytically. However, a truncation error is involved since the infinite series must be truncated to obtain numerical results. For comparison and validation purposes, the second order central finite difference method (FDM) is also implemented. The convergence analysis showed that CITT has a greater performance having no difficulties to obtain accurate results with very few terms in the solution summation. The FDM had convergence troubles specially for the positions near the center and for high concentration of heat generation in the center of the plate.

Keywords: heat conduction, classical integral transform technique, semianalytical formulation, heat generation

\section{NOMENCLATURE}

$\mathrm{Bi} \quad$ Biot number

$C_{p}$ fluid specific heat at constant pressure,

$$
\mathrm{J} /(\mathrm{kg} . \mathrm{K})
$$

erf error function

$g \quad$ volumetric heat generation, $\mathrm{W} / \mathrm{m}^{3}$

$\dot{g}^{*} \quad$ dimensionless heat generation

$\mathrm{G}$ total heat generation inputed in the plate

$i \quad$ direction $\mathrm{x}$ in the mesh

$j \quad$ direction $\mathrm{y}$ in the mesh

$k$ thermal conductivity, $\mathrm{W} /(\mathrm{m} . \mathrm{K})$

$N_{n}$ norms

\section{Greek symbols}

$\eta \quad$ dimensionless version of $\mathrm{r}$

$\theta$ dimensionless version of $\mathrm{T}$

$\bar{\theta}_{n} \quad$ transformed potential

$\lambda_{n}$ eigenvalues

$\xi \quad$ dimensionless version of $\mathrm{z}$

$\rho \quad$ material density, $\mathrm{kg} / \mathrm{m}^{3}$

$\sigma \quad$ parameter of the heat generation distribution

$\psi_{n} \quad$ eigenfunctions

$\Delta \eta \quad$ space between the nodes in direction y in the mesh

\section{$\Delta \xi \quad$ space between the nodes in direction $\mathrm{x}$ in the mesh}

\author{
Subscripts \\ n number of terms
}

\section{INTRODUCTION}

The development of high capacity components and sizes each time smaller are enabled due constant technological advancement of electronic components, specially microprocessors, integrated electronic circuits, data storage devices and memory (Knupp, 2010). The safe and efficient operation of these electronics components depend on circuit temperature, which should be within an acceptable limit. This limit can be maintained by dissipation of heat to the environment (Taposh, 2012). Indeed, it's hoped a new revolution in electronics with the improvement of development increasing of density of chips in a single printed circuit board and new materials usage. The consequence is the bigger difficulty at heat dissipation that is generated. Therefore, a bigger number of failures arise and it causes decrease of device useful life in case of this problem not to be solved from a proper and suitable manner.

Taking this into account, that is really important to develop devices that aid in heat dissipation. The easiest and most common way to face such kind of challenge is to lay such components at contact with parts of superficial area and thermal conductivity pretty high. 
Heat sinks, heat spreaders and convective foams have been designing to manage heat problems and control the temperature within safe range of operation in electronics cooling system. Heat sinks and Heat spreaders are composed for two parts; heat spreader which is the part of heat sink that enables the heat flux from source to extended surface and the extended surface providing high area to enable high heat flux. The limitation of extended surface of this components depend on the given volume. The effective heat coefficient of heat sink is proportional of available heat surface area. In general the space of these electronics components are installed is quite limited for extended surface. To solve this problem the use of conductive foam is great solution to replacement the surface areas given amount of area for convection heat transfer.

The use of conductive foams has great potential as extended surface area for thermal management and high surface area to volume ratio, that is why they are used in application where is impossible to use heat sinks or heat spreaders . Convective foams are made of aluminum, carbon or copper.

Classical analytical techniques applied to diffusion equation solution are capable to solve only problems with simple mathematical models. The necessity to obtain solutions of practical problems more complex each time, diversities solution techniques are deployed and target of frequent researches in many areas such numerical method, analytical method or numerical-analytical method.

Integral transforms are used by many authors since mathematics and physics applications until engineering science. The history has begun with laplace and Fourier transforms in nineteenth century. Laplace is successfully used for solving linear differential equations, meanwhile Fourier series may be used for theory of heat conduction and integrals with some applications (Chalhub and Sphaier, 2016).The British electrical engineer Oliver Heaviside (Oliver, 1894) was the professional who made the Laplace transform too popular by using it to solve ordinary differential equations in applications like electrical circuits and systems, and then developing modern operational calculus.

According to (Chalhub et al., 2013), the separation of variables method is an important technique of solution of diffusion equation, but its coverage is limited because the equation must obey certain restrictions to be separable. For this reason Classical Integral transform Technique-(CITT) was developed based on separation of variables having a methodology to solve a great variety of diffusion linear problems divided in seven classes. However according to (Cotta, 1998), the initial ideas was introduced by Koshlyakov in 1936, CITT gained power (Grimnberg, 1948) by applications in electric and magnetic problems. Some decades later, some authors like (Mikhailov and Osizik, 1984) and Tranter enhanced the idea. In 1974, Russia and some countries of East Europe afforded a great advancement in analytical methods deployment, such as Integral transforms (CITT). Meanwhile, USA and Europe gathered their endeavor to improve the development of numerical methods like Finite Differences, Finite element method and finite volume method.

In the realm of heat conduction, (Torabi and Zhang, 2014) solved by separation of variables and finite differences the case of cylindrical geometry with Neumann and Dirichlet boundary conditions considering dual-phase-lag model of heat conduction.

Rinaldi (2000) proposed an analytical formulation based on Fourier series to obtain the temperature distribution in integrated circuits.

Monteiro et al. (2009) used the generalized integral transform technique (GITT) to solve the hyperbolic heat conduction in a finite slab.

Sphaier and Cotta (2000, 2002) solved by integral transformation problems within irregular domains.

\section{PROBLEM FORMULATION}

The general heat conduction equation in classical two-dimensional cartesian coordinates with constant properties is given by:

$$
C_{p} \rho \frac{\partial T}{\partial t}=k\left(\frac{\partial T^{2}}{\partial x^{2}}+\frac{\partial T^{2}}{\partial y^{2}}\right)+\dot{g}
$$

Where $C_{p}$ is the specific heat, $\dot{g}$ is the volumetric heat generation, $k$ is the thermal conductivity and $\rho$ is the material density.

The problem to be considered in this work is the steady state heat conduction in a square plate with heat generation in the center of the plate with Robin boundary conditions, in a way that the symmetry allows us to solve only a quarter of plate. After nondimensionalization, the Eq. (1) can be rewritten as follows, with proper boundary conditions for the problem:

$$
\begin{aligned}
& \frac{\partial^{2} \theta}{\partial \xi^{2}}+\frac{\partial^{2} \theta}{\partial \eta^{2}}=\dot{g}^{*} \text { for } 0 \leq \xi \leq 1 \text { and } 0 \leq \eta \leq 1 \\
& \left.\frac{\partial \theta}{\partial \xi}\right|_{\xi=0}=0 ;\left.\frac{\partial \theta}{\partial \xi}\right|_{\xi=1}=\operatorname{Bi} \theta ;\left.\frac{\partial \theta}{\partial \eta}\right|_{\eta=0}=0 ;\left.\frac{\partial \theta}{\partial \eta}\right|_{\eta=1}=-\operatorname{Bi} \theta
\end{aligned}
$$

where $\theta, \xi$ and $\eta$ are nondimensional versions of $\mathrm{T}, \mathrm{x}$ and $\mathrm{y}$ respectively and the nondimensional heat generation is given by the function below: 


$$
\dot{g} *=\frac{G}{\sigma^{2} \pi} \exp \left[-\frac{\xi^{2}+\eta^{2}}{\sigma^{2}}\right]
$$

where $\mathrm{G}$ is the total heat generation inputed in the plate and $\sigma(0 \leq \sigma \leq 0.5)$ is a parameter of the heat generation distribution. When $\sigma$ approaches to zero, the heat generation is more concentrated in the center of plate. On the other hand, when $\sigma$ is lager, the generation is more spread out.

\section{Classical Integral Transform Technique (CITT)}

Classical integral transform technique is the extension of variables separation method, and consist to establishment of inverse-transformed pair of each potential in term of orthogonal eigenfunction bases, that are obtained from auxiliary problems of eigenvalue appropriately chosen. The classical integral transformation of the original differential equation is obtained by adequate operators that reduce the order of the partial derivatives of the second order. Making use of transformation function, one can obtain an infinite uncoupled system of ordinary differential equations for transformed potential, that can be solved analytically. From the inversion formula, one can obtain the analytical solution of each potential (Chalhub, 2011).

In order to solve the temperature distribution problem given by Eq. (2) with CITT, appropriate Helmhotz eigenvalue problem is taken:

$$
\begin{gathered}
\frac{\mathrm{d} \psi_{n}}{\mathrm{~d} \xi^{2}}+\lambda_{n}^{2} \psi_{n}=0 \\
\left.\frac{\mathrm{d} \psi_{n}}{\mathrm{~d} \xi}\right|_{\xi=0}=0 ;\left.\frac{\mathrm{d} \psi_{n}}{\mathrm{~d} \xi}\right|_{\xi=1}=-\mathrm{Bi} \psi_{n}
\end{gathered}
$$

where $\psi_{n}$ are eigenfunctions and $\lambda_{n}$ are eigenvalues, and the solution to the Helmhotz eigenvalue problem is given by:

$$
\psi_{n}(\xi)=\cos \left(\lambda_{n} \xi\right)
$$

The eigenvalues are calculated from the transcendental equation below:

$$
\tan \left(\lambda_{n}\right)=\frac{\mathrm{Bi}}{\lambda_{n}} \text { for } n=1,2,3 \ldots
$$

The transformation pair is defined by:

$$
\text { Transformation } \Rightarrow \bar{\theta}_{n}(\eta)=\int_{0}^{1} \theta(\xi, \eta) \psi_{n}(\xi) \mathrm{d} \xi
$$

$$
\text { Inversion } \Rightarrow \theta(\xi, \eta)=\sum_{n=1}^{\infty} \frac{\overline{\theta_{n}}(\eta) \psi_{n}(\xi)}{N_{n}}
$$

where the norms $N_{n}$ are defined by:

$$
N_{n}=\int_{0}^{1} \psi_{n}^{2} \mathrm{~d} \xi=\frac{1}{4}\left[\frac{\sin \left(2 \lambda_{n}\right)}{\lambda_{n}}+2\right]
$$

The integral transformation of the governing differential equation is derived by applying the operator $\int_{0}^{1}(\mathrm{o}) \psi_{n} \mathrm{~d} \xi$ on Eqs. (2) leading to the following transformed equation:

$$
\begin{gathered}
-\lambda_{n}^{2} \bar{\theta}_{n}+\frac{\mathrm{d}^{2} \bar{\theta}_{n}}{\mathrm{~d} \xi^{2}}=-\bar{g}_{n}^{*}(\eta) \\
\left.\frac{\mathrm{d} \bar{\theta}_{n}}{\mathrm{~d} \eta}\right|_{\eta=0}=0 ;\left.\frac{\mathrm{d} \bar{\theta}_{n}}{\mathrm{~d} \eta}\right|_{\eta=1}=-\mathrm{Bi} \bar{\theta}_{n}(1)
\end{gathered}
$$

The transformed Eq. (11) admits the following analytical solution:

$$
\bar{\theta}_{n}(\eta)=\left\{\frac{\mathrm{Ge}^{(-\eta-1) \lambda_{n}}\left(\begin{array}{l}
\operatorname{erf}\left(\frac{1}{\sigma}-\frac{1}{2} i \sigma \lambda_{n}\right) \\
+\operatorname{erf}\left(\frac{1}{\sigma}+i \sigma \lambda_{n}\right)
\end{array}\right)}{32 \lambda_{n}\left(\begin{array}{l}
\operatorname{Bicosh}\left(\lambda_{n}\right)+ \\
\lambda_{n} \sinh \left(\lambda_{n}\right)
\end{array}\right)}\right\}
$$

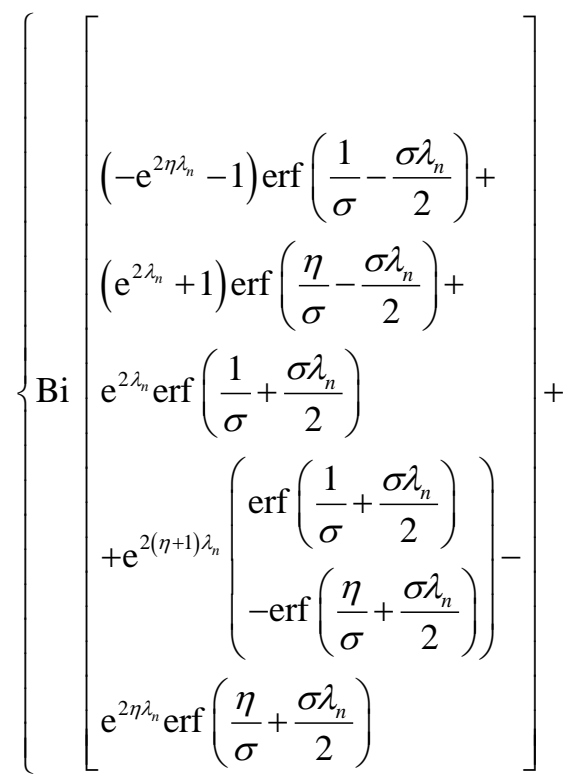




$$
\left.\left[\begin{array}{c}
\left(\mathrm{e}^{2 \eta \lambda_{n}}+1\right) \operatorname{erf}\left(\frac{1}{\sigma}-\frac{\sigma \lambda_{n}}{2}\right)+ \\
\left(\mathrm{e}^{2 \lambda_{n}}-1\right) \operatorname{erf}\left(\frac{\eta}{\sigma}-\frac{\sigma \lambda_{n}}{2}\right) \\
+\mathrm{e}^{2 \lambda_{n}} \operatorname{erf}\left(\frac{1}{\sigma}+\frac{\sigma \lambda_{n}}{2}\right)+ \\
\mathrm{e}^{2(\eta+1) \lambda_{n}}\left(\begin{array}{c}
\operatorname{erf}\left(\frac{1}{\sigma}+\frac{\sigma \lambda_{n}}{2}\right) \\
-\mathrm{erf}\left(\frac{\eta}{\sigma}+\frac{\sigma \lambda_{n}}{2}\right)
\end{array}\right.
\end{array}\right]+\lambda\right\}
$$

where erf is the error function.

In order to obtain the final solution for $\theta$, the inversion formula must be used and the summation must be truncated to a finite value $\left(n_{\max }\right)$.

\section{Finite Differences Method(FDM)}

The classical second order finite differences method was also used in this work for the purpose to compare and validate the results. The discretized form of the governing Eq. (2) valid inside the domain is given by:

$$
\frac{\theta_{i, j-1}-2 \theta_{i, j}+\theta_{i, j+1}}{\Delta \eta^{2}}+\frac{\theta_{i-1, j}-2 \theta_{i, j}+\theta_{i+1, j}}{\Delta \xi^{2}}=
$$

$-\dot{g}^{*}{ }_{i, j}$ for $2 \leq i \leq i_{\max -1}$ and $2 \leq \mathrm{j} \leq \mathrm{j}_{\max -1}$

The discretized heat generation function is:

$$
\dot{g}^{*}{ }_{i, j}=\dot{g}^{*}((i-1) \Delta \xi,(j-1) \Delta \eta)
$$

For boundary conditions, the following discretization applies:

For $2 \leq i \leq i_{\max -1}$ and $j=j_{\max }$ :

$$
\begin{gathered}
\frac{-2 \mathrm{Bi} \Delta \eta \theta_{\mathrm{i}, \mathrm{j}}+2 \theta_{i, j-1}-2 \theta_{i, j}}{\Delta \eta^{2}}+ \\
\frac{\theta_{\mathrm{i}-1, \mathrm{j}}-2 \theta_{i, j}+\theta_{i+1, j}}{\Delta \xi^{2}}=-\dot{g}^{*}{ }_{i, j}
\end{gathered}
$$

For $2 \leq i \leq i_{\max -1}$ and $\mathrm{j}=1$ :

$$
\frac{2 \theta_{\mathrm{i}, \mathrm{j}+1}-2 \theta_{i, j}}{\Delta \eta^{2}}+\frac{\theta_{\mathrm{i}-1, \mathrm{j}}-2 \theta_{i, j}+\theta_{\mathrm{i}+1, \mathrm{j}}}{\Delta \xi^{2}}=-\dot{g}^{*}{ }_{i, j}
$$

For $i=i_{\max }$ and $2 \leq j \leq j_{\max -1}$ :

$$
\begin{aligned}
& \frac{-2 \mathrm{Bi} \Delta \xi \theta_{i, j}+2 \theta_{i-1, j}-2 \theta_{i, j}}{\Delta \xi^{2}}+ \\
& \frac{\theta_{\mathrm{i}, \mathrm{j}-1}-2 \theta_{i, j}+\theta_{i, j+1}}{\Delta \eta^{2}}=-\dot{g}^{*}{ }_{i, j}
\end{aligned}
$$

For $i=1$ and $2 \leq j \leq j_{\max -1}$ :

$$
\begin{aligned}
& \frac{\theta_{\mathrm{i}, \mathrm{j}-1}-2 \theta_{i, j}+\theta_{i, j+1}}{\Delta \eta^{2}}+ \\
& \frac{2 \theta_{\mathrm{i}+1, \mathrm{j}}-2 \theta_{i, j}}{\Delta \xi^{2}}=-\dot{g}^{*}{ }_{i, j}
\end{aligned}
$$

For $i=i_{\max }$ and $j=j_{\max }$ :

$$
\begin{aligned}
& \frac{-2 \mathrm{Bi} \Delta \eta \theta_{\mathrm{i}, \mathrm{j}}+2 \theta_{i, j-1}-2 \theta_{i, j}}{\Delta \eta^{2}}+ \\
& \frac{-2 \mathrm{Bi} \Delta \xi \theta_{\mathrm{i}, \mathrm{j}}+2 \theta_{i-1, j}-2 \theta_{i, j}}{\Delta \xi^{2}}=-\dot{g}^{*}{ }_{i, j}
\end{aligned}
$$

For $i=1$ and $j=j_{\max }$ :

$$
\begin{aligned}
& \frac{-2 \mathrm{Bi} \Delta \eta \theta_{\mathrm{i}, \mathrm{j}}+2 \theta_{i, j-1}-2 \theta_{i, j}}{\Delta \eta^{2}}+ \\
& \frac{2 \theta_{i+1, j}-2 \theta_{i, j}}{\Delta \xi^{2}}=-\dot{g}^{*}{ }_{i, j}
\end{aligned}
$$

For $i=i_{\max }$ and $j=1$ :

$$
\begin{aligned}
& \frac{-2 \mathrm{Bi} \Delta \xi \theta_{i, j}+2 \theta_{i-1, j}-2 \theta_{i, j}}{\Delta \xi^{2}}+ \\
& \frac{2 \theta_{i, j+1}-2 \theta_{i, j}}{\Delta \eta^{2}}=-\dot{g}^{*}{ }_{i, j}
\end{aligned}
$$

For $i=1$ and $j=1$ :

$$
\frac{2 \theta_{i, j+1}-2 \theta_{i, j}}{\Delta \eta^{2}}+\frac{2 \theta_{i+1, j}-2 \theta_{i, j}}{\Delta \xi^{2}}=-\dot{g}^{*}{ }_{i, j}
$$

The algebraic system of equations generated by FDM formulation is solved using the Mathematica (Wolfram, 2003) subroutine FindRoot.

\section{RESULTS AND DISCUSSION}

After describing the problem formulation and 
solution methodology, numerical results are presented in this section. The first set of results are dedicated to exam the solution convergence using two mathematical models for the proposed problem solution: Classical Integral Transforms Technique and Finite Differences Method. Results showed in the following tables have been analyzed for different Biot Numbers $(\mathrm{Bi}=0.01 ; 0.1 ; 1 ; 10 ; 100)$, different heat generation distribution parameters $(\sigma=0.01$; 0.2 ) and the heat internal generation has been fixed as $\mathrm{G}=1$.

The mathematical tool used for such simulation was rather known software in engineering, mathematics and physical science: Wolfram Mathematica 10.0. Its provides a very optimal symbolic language, that is totally different from most usual programs, we can say Mathematica is able to work with mathematical expressions meanwhile presents a interpretative language. More advantages are related to the fact that it gets to join a symbolic manipulation, a flexible language, numerical calculation accomplishment in its work platform, besides of setting up graphics and tables in only one spot.

For illustration purposes, Figure 1 shows the plot of the heat generation function for $\eta=0, \mathrm{G}=1$ and for different $\sigma$ 's. It is possible to see that for smaller $\sigma$ 's, the generation is more concentrated in the center of the plate, while for larger $\sigma$ 's the generation is more evenly distributed in the domain. It should be highlighted that for any $\sigma$ varying between 0 and 0.5 , the integration of the heat generation function in the domain $(0 \leq \xi \leq 1$ and $0 \leq \eta \leq 1$ ) gives the result of G/4, which represents one fourth of the total energy inputed in the plate. In other words:

$$
\int_{0}^{1} \int_{0}^{1} \dot{g^{*}}(\xi, \eta) \mathrm{dxdy}=\frac{\mathrm{G}}{4}
$$

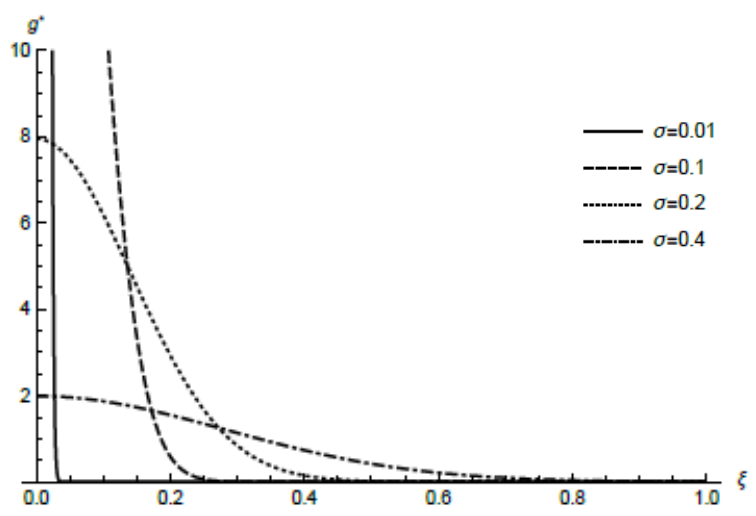

Figure 1. Heat generation function $\dot{g}$ * plotted for $\eta$ $=0, \mathrm{G}=1$ and for different parameters $\sigma$.
Table 1 shows the convergence analysis of temperature with heat generation distribution $\sigma=$ 0.01 for 5 different positions on the plate $(\xi=0$; $0.25 ; 0.5 ; 0.75 ; 0.9$ and $\eta=0 ; 0.25 ; 0.5 ; 0.75 ; 0.9$ ) and different Biot values $(\mathrm{Bi}=0.01 ; 0.1 ; 1 ; 10 ; 100)$. On Tab. 1, the technique used to the solution of problem has been CITT. It can be noticed that close to the heat source, represented by point ( $\xi=0, \eta=0$ ), it's necessary slightly more terms to achieve six significant figures convergence. On the other hand, close to the plate corner, represented on Tab. 1 by the column ( $\xi=0.9 ; \eta=0.9$ ), the temperature converges faster, one can see that it only requires four terms in the summation of CITT. This tendency of the convergence occurs for all Biot numbers when $\sigma$ is fixed in 0.01. It is also possible to see that as Biot number increases the converge rate improves.

Table 2 shows the same results as Tab. 1, but with $\sigma=0.2$. The heat generation for this case is more distributed in the domain. It can be seen that the convergence behavior is very similar to Tab. 1 , the convergence is slightly better at corner of the plate and the same comments can be made on this table. Comparing behaviors of both tables, it's noticed that the temperature gradient in the Tab. 1 is bigger due to the heat generation to be more concentrated in the center. One can observe that, overall the CITT solution requires very small truncation orders $\left(n_{\max }\right)$, as seen on tables, for a six digit convergence it is necessary only up to six terms for all positions analyzed.

Table 1. Temperature convergence solved by CITT for $\sigma=0.01$ and different Biot numbers.

\begin{tabular}{|c|c|c|c|c|c|}
\hline$n_{\max }$ & $\begin{array}{l}\xi=0 \\
\eta=0\end{array}$ & $\begin{array}{l}\xi=0.25 \\
\eta=0.25\end{array}$ & $\begin{array}{l}\xi=0.5 \\
\eta=0.5\end{array}$ & $\begin{array}{l}\xi=0.75 \\
\eta=0.75\end{array}$ & $\begin{array}{l}\xi=0.9 \\
\eta=0.9\end{array}$ \\
\hline \multicolumn{6}{|c|}{$\mathrm{Bi}=0.01$} \\
\hline 2 & 12.8639 & 12.6976 & 12.5833 & 12.5080 & 12.4697 \\
\hline 4 & 12.9908 & 12.6941 & 12.5798 & 12.5080 & 12.4700 \\
\hline 6 & 13.0278 & 12.6924 & 12.5799 & 12.5080 & 12.4700 \\
\hline 8 & 13.0278 & 12.6924 & 12.5799 & 12.5080 & 12.4700 \\
\hline 10 & 13.0278 & 12.6924 & 12.5799 & 12.5080 & 4700 \\
\hline \multicolumn{6}{|c|}{$\mathrm{Bi}=0.1$} \\
\hline 2 & 1.61388 & 1.44716 & 1.33288 & 1.25829 & 1.22094 \\
\hline 4 & 1.74036 & 1.44355 & 1.32948 & 1.25833 & 1.22122 \\
\hline 6 & 1.77728 & 1.44183 & 1.32956 & 1.25833 & 1.22122 \\
\hline 8 & 1.77728 & 1.44183 & 1.32956 & 1.25833 & 1.22122 \\
\hline 10 & 1.77728 & 1.44183 & 1.32956 & 1.25833 & 1.22122 \\
\hline \multicolumn{6}{|c|}{$\mathrm{Bi}=1$} \\
\hline 2 & 0.490375 & 0.318809 & 0.204931 & 0.135587 & 0.104541 \\
\hline 4 & 0.612620 & 0.314748 & 0.201994 & 0.135686 & 0.104779 \\
\hline 6 & 0.649116 & 0.313083 & 0.201994 & 0.135683 & 0.104779 \\
\hline 8 & 0.649116 & 0.313083 & 0.201994 & 0.135683 & 0.104779 \\
\hline 10 & 0.649116 & 0.313083 & 0.201994 & 0.135683 & 0.104779 \\
\hline \multicolumn{6}{|c|}{$\mathrm{Bi}=10$} \\
\hline 2 & 0.394293 & 0.199961 & 0.0858813 & 0.0276721 & 0.00898337 \\
\hline 4 & 0.500599 & 0.194439 & 0.0844319 & 0.0278626 & 0.00906979 \\
\hline 6 & 0.534390 & 0.193114 & 0.0844787 & 0.0278610 & 0.00906999 \\
\hline 8 & 0.534390 & 0.193114 & 0.0844787 & 0.0278610 & 0.00906999 \\
\hline 10 & 0.534390 & 0.193114 & 0.0844787 & 0.0278610 & 0.00906999 \\
\hline \multicolumn{6}{|c|}{$\mathrm{Bi}=100$} \\
\hline 2 & 2441 & 0.186385 & 0.0724133 & & 0.0032 \\
\hline
\end{tabular}




\begin{tabular}{|c|c|c|c|c|c|}
\hline 4 & 0.496000 & 0.180332 & $\begin{array}{l}0.0715878 \\
\end{array}$ & 0.0181598 & 0.00324488 \\
\hline 6 & 0.496000 & 0.180332 & 0.0715878 & 0.0181598 & 0.00324488 \\
\hline 8 & 0.496000 & 0.180332 & 0.0715878 & 0.0181598 & \begin{tabular}{|c|}
0.00324488 \\
\end{tabular} \\
\hline 10 & 0.496000 & 0.180332 & \begin{tabular}{|l|}
0.0715878 \\
\end{tabular} & 0.0181598 & 0.00324488 \\
\hline \multicolumn{6}{|c|}{$\begin{array}{l}\text { Table 2. Temperature convergence solved by CITT } \\
\text { for } \sigma=0.2 \text { and different Biot numbers. }\end{array}$} \\
\hline$n_{\text {max }}$ & $\begin{array}{l}\xi=0 \\
\eta=0\end{array}$ & $\begin{array}{l}\xi=0.25 \\
\eta=0.25\end{array}$ & $\begin{array}{l}\xi=0.5 \\
\eta=0.5\end{array}$ & $\begin{array}{l}\xi=0.75 \\
\eta=0.75\end{array}$ & $\begin{array}{l}\xi=0.9 \\
\eta=0.9\end{array}$ \\
\hline \multicolumn{6}{|c|}{$\mathrm{Bi}=0.01$} \\
\hline 2 & 12.7852 & 12.6951 & 12.5833 & 12.5080 & 12.4697 \\
\hline 4 & 12.8277 & 12.6924 & 12.5798 & 12.5 & 12.4700 \\
\hline 6 & 12.8277 & 12.6913 & 12.5799 & 12.5080 & 12.4700 \\
\hline 8 & 12.8277 & 12.6913 & 12.5799 & 12.5080 & 12.4700 \\
\hline 10 & 12.8277 & 12.6913 & 12.5799 & 12.5080 & 12.4700 \\
\hline \multicolumn{6}{|c|}{$\mathrm{Bi}=0.1$} \\
\hline 2 & 1.53496 & 1.44462 & 1.33288 & 1.25829 & 1.22094 \\
\hline 4 & 1.57413 & 1.44 & 1.32 & & 1.22122 \\
\hline 6 & 1.57713 & 1.44079 & 1.32955 & 1.25 & 1.22122 \\
\hline 8 & 1.57713 & 1.44079 & 1.32955 & 1.25833 & 1.22122 \\
\hline 10 & 1.57713 & 1.44079 & 1.32955 & 1.25833 & 1.22122 \\
\hline \multicolumn{6}{|c|}{$\mathrm{Bi}=1$} \\
\hline 2 & 0.408705 & 0.316242 & 0.204928 & 0.135587 & 0.104541 \\
\hline 4 & 0.445450 & 0.313039 & 0.201925 & 0.135686 & 0.104779 \\
\hline 6 & 0.448347 & 0.312 & 0.20 & 583 & \begin{tabular}{|l|}
0.104779 \\
\end{tabular} \\
\hline 8 & 0.448347 & 0.312 & 0.201994 & 0.13 & 0.104779 \\
\hline 10 & 0.448347 & 0.312046 & 0.201994 & 0.135683 & 0.104779 \\
\hline \multicolumn{6}{|c|}{$\mathrm{Bi}=10$} \\
\hline 2 & 0.299442 & 0.197290 & 0.0858795 & 0.0276721 & 0.0089833 \\
\hline 4 & 0.326318 & 0.192891 & 0.0844329 & 0.0278626 & 0.0090697 \\
\hline 6 & 0.328569 & 0.192147 & 0.0844783 & 0.0278610 & 0.0090699 \\
\hline 8 & 0.328569 & 0.192147 & 0.0844783 & 0.0278610 & 0.0090699 \\
\hline 10 & 0.328569 & 0.192147 & 0.0844783 & 0.0278610 & 0.0090699 \\
\hline \multicolumn{6}{|c|}{$\mathrm{Bi}=100$} \\
\hline 2 & 0.290554 & 0.183693 & \begin{tabular}{|l|}
0.0724124 \\
\end{tabular} & 0.0179988 & 0.0032110 \\
\hline 4 & 0.313458 & 0.178964 & 0.0715883 & 0.0181598 & 0.0032448 \\
\hline 6 & 0.313458 & 0.178964 & 0.0715883 & 0.0181598 & 0.0032448 \\
\hline 8 & 0.313458 & 0.178964 & 0.0715883 & 0.0181598 & 0.0032448 \\
\hline 10 & 0.313458 & 0.178964 & 0.0715883 & 0.0181598 & 0.0032448 \\
\hline
\end{tabular}

On Tab. 3, it is introduced results of temperature convergence analysis for the presented problem, using finite difference method (FDM). This table presents results for the same cases as Tab. 1 . In finite difference (FDM) for these cases with $\sigma=$ 0.01 the temperature convergence occurs to mesh 200 $\times 200$ in most points analyzed, it is noticed that when Biot number increases the convergence rate deteriorates. In addition is showed in the Tab. 3 the converged temperature achieved by (CITT) for each position and mesh $200 \times 200$ is very refined taking amount of computational memory. Making the analysis closer to the center, in position ( $\xi=0 ; \eta=0$ ) the temperature convergence of any significant figures is not achieved even with a mesh of $200 \times$ 200. The same pattern from CITT is observed here, as we approach the boundary, the convergence rate improves and for some cases it is possible to obtain the convergence of six digits with a mesh of $200 \times$ 200.

Table 3. Temperature convergence solved by FDM for $\sigma=0.01$ and different Biot numbers.

\begin{tabular}{|c|c|c|c|c|c|}
\hline$i_{\max }$ & $\xi=0 ;$ & $\xi=0.25 ;$ & $\xi=0.5 ;$ & $\xi=0.75 ;$ & $\xi=0.9 ;$ \\
$j_{\max }^{=}$ & $\eta=0$ & $\eta=0.25$ & $\eta=0.5$ & $\eta=0.75$ & $\eta=0.9$ \\
\hline
\end{tabular}

\begin{tabular}{|c|c|c|c|c|c|}
\hline \multicolumn{6}{|c|}{$\mathrm{Bi}=0.01$} \\
\hline 12 & 346.349 & 333.866 & \begin{tabular}{|l|}
330.933 \\
\end{tabular} & 329.042 & 328.041 \\
\hline 25 & 73.4426 & 70.1381 & 69.5192 & 69.1218 & 68.9118 \\
\hline 50 & 18.8695 & 17.8878 & 17.7298 & 17.6285 & 17.5749 \\
\hline 100 & 13.3336 & 12.6952 & 12.5831 & 12.5111 & 12.4731 \\
\hline 200 & 13.3108 & 12.6920 & 12.5799 & 12.5080 & 12.4700 \\
\hline $\begin{array}{l}\text { Conv. } \\
\text { CITT }\end{array}$ & 13.0278 & 12.6924 & 12.5799 & 12.5080 & 12.4700 \\
\hline \multicolumn{6}{|c|}{$\mathrm{Bi}=0.1$} \\
\hline 12 & 50.3850 & 37.9021 & 34.9740 & 33.1012 & 32.1242 \\
\hline 25 & 11.2697 & 7.96524 & 7.34729 & 6.95373 & 6.74863 \\
\hline 50 & 3.01324 & 2.03157 & 1.87384 & 1.77346 & 1.72115 \\
\hline 100 & 2.08023 & 1.44186 & 1.32989 & 1.25864 & 1.22152 \\
\hline 200 & 2.06023 & 1.44150 & 955 & 1.25833 & 1.22122 \\
\hline $\begin{array}{l}\text { Conv. } \\
\text { CITT }\end{array}$ & 1.77728 & & & & 1.22122 \\
\hline \multicolumn{6}{|c|}{$\mathrm{Bi}=1$} \\
\hline 12 & 20.6877 & 8.207000 & 5.31020 & 3.56777 & 2.75480 \\
\hline 25 & 5.03157 & 1.72 & 1.11609 & 0.749739 & 0.578962 \\
\hline 50 & 1.42231 & 0.440757 & 0.284673 & 1224 & 17669 \\
\hline 100 & 0.951133 & 0.312839 & 0.202041 & 0.135717 & 0.104805 \\
\hline 200 & 0.931424 & 0.312767 & 0.201991 & 0.135683 & 0.104779 \\
\hline $\begin{array}{l}\text { Conv. } \\
\text { CITT }\end{array}$ & 0.649116 & 0.313083 & 0.201994 & 0.135683 & 0.104779 \\
\hline \multicolumn{6}{|c|}{$\mathrm{Bi}=10$} \\
\hline 12 & 17.5264 & 5.05024 & 2.21584 & 0.730827 & 0.237865 \\
\hline 25 & 4.36825 & 1.06508 & 0.466533 & 0.153871 & 0.0500902 \\
\hline 50 & 1.25318 & 0.271846 & 0.119039 & 0.0392610 & 0.0127810 \\
\hline 100 & 0.831105 & 0.192966 & 0.0844930 & 0.0278672 & 0.0090719 \\
\hline 200 & 0.811428 & 0.192927 & 0.0844741 & 0.0278609 & 0.0090699 \\
\hline $\begin{array}{l}\text { Conv. } \\
\text { CITT }\end{array}$ & 0.534390 & 0.193114 & 0.0844787 & 0.0278610 & 0.0090699 \\
\hline \multicolumn{6}{|c|}{$\mathrm{Bi}=100$} \\
\hline 12 & 17.1649 & 4.69015 & 1.87646 & 0.475624 & 0.0849566 \\
\hline 25 & 4.29255 & 0.989672 & 0.395388 & 0.100258 & 0.0179144 \\
\hline 50 & 1.23389 & 0.252626 & 0.100902 & 0.0255877 & 0.0045723 \\
\hline 100 & 0.817414 & 0.179327 & 0.0716224 & 0.0181630 & 0.0032456 \\
\hline 200 & 0.797741 & 0.179292 & 0.0716071 & 0.0181592 & 0.0032449 \\
\hline $\begin{array}{l}\text { Conv. } \\
\text { CITT }\end{array}$ & 0.496000 & 0.180332 & 0.0715878 & 0.0181598 & 0.0032448 \\
\hline
\end{tabular}

Table 4 displays similar results as Tab. 2, but computed by FDM. It can be seen that the overall convergence is better than 3 , this can be explained by better heat generation distribution throughout to plate. The convergence is reached in most points of the plate especially close to the boundary, but the six digits convergence in the center is not reached for any Biot. As mentioned for the Tab. 3 the convergence rate worsens when Biot number increases.

Table 4. Temperature convergence solved by FDM for $\sigma=0.2$ and different Biot numbers.

\begin{tabular}{|c|c|c|c|c|c|}
\hline $\begin{array}{c}i_{\max } \\
j_{\max }\end{array}$ & $\xi=0 ;$ & $\xi=0.25 ;$ & $\xi=0.5 ;$ & $\xi=0.75 ;$ & $\xi=0.9 ;$ \\
\hline \multicolumn{7}{|c|}{$\mathrm{Bi}=0.01$} \\
\hline 12 & 12.8332 & 12.6909 & 12.5799 & 12.5080 & 12.4699 \\
\hline 25 & 12.8297 & 12.6912 & 12.5799 & 12.5080 & 12.4700 \\
\hline 50 & 12.8290 & 12.6911 & 12.5799 & 12.5080 & 12.4700 \\
\hline 100 & 12.8288 & 12.6911 & 12.5799 & 12.5080 & 12.4700 \\
\hline 200 & 12.8288 & 12.6911 & 12.5799 & 12.5080 & 12.4700 \\
\hline $\begin{array}{c}\text { Conv. } \\
\text { CITT }\end{array}$ & 12.8277 & 12.6913 & 12.5799 & 12.5080 & 12.4700 \\
\hline \multicolumn{7}{|c|}{$\mathrm{Bi}=0.1$} \\
\hline 12 & 1.58265 & 1.44035 & 1.32952 & 1.25830 & 1.22116 \\
\hline 25 & 1.57913 & 1.44064 & 1.32955 & 1.25832 & 1.22121 \\
\hline 50 & 1.57843 & 1.44061 & 1.32955 & 1.25833 & 1.22122 \\
\hline
\end{tabular}




\begin{tabular}{|c|c|c|c|c|c|}
\hline 100 & 1.57827 & 1.44061 & 1.32955 & 1.25833 & 1.22122 \\
\hline 200 & 1.57823 & 1.44061 & 1.32955 & 1.25833 & 1.22122 \\
\hline $\begin{array}{l}\text { Conv. } \\
\text { CITT }\end{array}$ & 1.57713 & 1.44079 & 1.32955 & 1.25833 & 1.22122 \\
\hline \multicolumn{6}{|c|}{$\mathrm{Bi}=1$} \\
\hline 12 & 0.453755 & 0.311540 & 0.201908 & 0.135636 & 0.104728 \\
\hline 25 & 0.450298 & 0.311896 & 0.201973 & 0.135672 & 0.104768 \\
\hline 50 & 0.449620 & 0.311875 & 0.201987 & 0.135681 & 0.104776 \\
\hline 100 & 0.449460 & 0.311881 & 0.201991 & 0.135683 & 0.104778 \\
\hline 200 & 0.449421 & 0.311883 & 0.201992 & 0.135683 & 0.104779 \\
\hline $\begin{array}{l}\text { Conv. } \\
\text { CITT }\end{array}$ & 0.448347 & 0.312046 & 0.201994 & 0.135683 & 0.104779 \\
\hline \multicolumn{6}{|c|}{$\mathrm{Bi}=10$} \\
\hline 12 & 0.333590 & 0.191544 & 0.0842830 & 0.0277909 & 0.00904503 \\
\hline 25 & 0.330266 & 0.192023 & 0.0844331 & 0.0278459 & 0.00906475 \\
\hline 50 & 0.329616 & 0.192027 & 0.0844648 & 0.0278575 & 0.00906873 \\
\hline 100 & 0.329461 & 0.192039 & 0.0844724 & 0.0278603 & 0.00906968 \\
\hline 200 & 0.329424 & 0.192042 & 0.0844742 & 0.0278609 & 0.00906991 \\
\hline $\begin{array}{l}\text { Conv. } \\
\text { CITT }\end{array}$ & 0.328569 & 0.192147 & 0.0844783 & 0.0278610 & 0.00906999 \\
\hline \multicolumn{6}{|c|}{$\mathrm{Bi}=100$} \\
\hline 12 & 0.319846 & 0.177857 & 0.0713824 & 0.0180885 & 0.00323093 \\
\hline 25 & 0.316568 & 0.178377 & 0.0715591 & 0.0181441 & 0.00324203 \\
\hline 50 & 0.315926 & 0.178389 & 0.0715962 & 0.0181557 & 0.00324428 \\
\hline 100 & 0.315774 & 0.178403 & 0.0716050 & 0.0181585 & 0.00324481 \\
\hline 200 & 0.315737 & 0.178407 & 0.0716072 & 0.0181592 & 0.00324494 \\
\hline $\begin{array}{l}\text { Conv. } \\
\text { CITT }\end{array}$ & 0.313458 & 0.178964 & 0.0715883 & 0.0181598 & 0.00324488 \\
\hline
\end{tabular}

In general, one can see that CITT has no difficulties obtaining the temperature with six digits accuracy for cases showed in this work with a truncation order no more than six for all computed cases. It seems that CITT convergence does not depend on $\sigma$ and it presents good results overall. On the other hand, FDM has troubles computing the results for smaller $\sigma$ 's.

\section{CONCLUSIONS}

In the present work, it was proposed a semianalytical formulation based on classical integral transform technique (CITT) for the solution of the heat conduction with heat generation in a plate. For compared proposes, it was also developed a numerical formulation based on second order central finite differences method (FDM). The analysis of heat generation distribution was considered in this problem as well as the Biot numbers for the boundary conditions.

The convergence analysis showed that CITT has a greater performance having no difficulties to obtain six digits accuracy with very few terms in the solution summation. The FDM had convergence troubles specially for the positions near the center and for lower $\sigma$ 's, not even converging with a mesh of $200 \times 200$.

Finally, the proposed semi-analytical approach has shown to be a good alternative method for this kind of problem.

\section{ACKNOWLEDGEMENTS}

The authors would like to acknowledge the financial support provided by FAPERJ, CAPES and
Universidade do Estado do Rio de Janeiro.

\section{REFERENCES}

Chalhub, D. J. N. M., 2011, Desenvolvimento de Soluções para Problemas de Advecção-Difusão Combinando Transformação Integral e Métodos Discretos, Master Thesis, Universidade Federal Fluminense, Niterói-RJ. (in Portuguese)

Chalhub, D. J. N. M., Sphaier, L. A., and Alves, L. S. de B., 2013, Integral Transform Solution of Convective Heat Transfer Problems using Upwind Approximations, Numerical Heat Transfer, Part B, Vol. 63, pp. 167-187.

Chalhub, D. J. N. M., and Sphaier, L. A., 2016, Integral Transform Solution for ThermallyDeveloping Slip-Flow within Isothermal Parallel Plates, Computational Thermal Sciences, Universidade Federal Fluminense, Niterói-RJ.

Cotta, R. M., 1998, The Integral Transform Method in Thermal and Fluids Science and Engineering, Begell House, Inc.

Grimnberg, G., 1948, Selected Problems of Mathematical Theory of Electrical and Magnetic Effects, Nauk.

Knupp, D. C., 2010, Análise TeóticoExperimental de Transferência de Calor em Nanocompósitos via Transformação Integral e Termografia por Infravermelho, Master Thesis, Universidade Federal do Rio de Janeiro, Rio de Janeiro.

Mikhailov, M. D., and Osizik, M. N., 1984, Unified Analysis and Solutions of Heat and Mass Diffusion, John Wiley \& Sons, New York.

Monteiro, E. R., Macêdo, E. N., Quaresma, J. N., and Cotta, R. M., 2009, Integral Transform Solution for Hyperbolic Heat Conduction in a Finite Slab, International Communications in Heat and Mass Transfer, Vol. 36, pp. 297-303.

Oliver, H., 1894, Electrical Papers, Vol.1 and Vol. 2., New York and London: Macmillan and Co.

Rinaldi, N., 2000, Thermal Analysis of SolidState Devices and Circuits: an Analytical Approach, Solid-State Electronics, Vol. 44, pp. 1789-1798.

Sphaier, L. A., and Cotta, R. M., 2000, Integral Transform Analysis of Multidimensional Eigenvalue Problems within Irregular Domains, Numerical Heat Transfer, Part B: Fundamentals, Vol. 38, pp. 157175.

Sphaier, L. A., and Cotta, R. M., 2002, Analytical and Hybrid Solutions of Diffusion Problems within Arbitrarily Shaped Regions via Integral Transforms, Computational Mechanics, Vol. 29, pp. 265-276.

Taposh, R. M., 2012, Optimization of Heat Spreader, Master Thesis, Russ College of Engineering and Technology of Ohio University.

Torabi, M., and Zhang, K., 2014, MultiDimensional Dual-Phase-Lag Heat Conduction in Cylindrical Coordinates: Analytical and Numerical Solutions, International Journal of Heat and Mass 
Transfer, Vol. 78, pp. 960-966.

Wolfram, S., 2003, The Mathematica Book, Wolfram Media/Cambridge University Press, New York/Champaign, IL, 5th edition. 